\title{
Reestenosis de la arteria coronaria descendente anterior en mujeres diabéticas
}

\author{
Franklin E. Choles, Tania P. Rodríguez*, Leidy J. Plata y Karen L. Sampayo
}

Facultad de Medicina, Universidad del Magdalena, Santa Marta, Colombia

Recibido el 29 de diciembre de 2015; aceptado el 25 de abril de 2016

Disponible en Internet el 18 de julio de 2016

\section{PALABRAS CLAVE \\ Cirugía de revascularización coronaria; Diabetes mellitus; \\ Stent; Intervencionismo coronario percutáneo; Reestenosis}

\section{KEYWORDS \\ Coronary revascularization surgery; Diabetes mellitus; Stent;}

\begin{abstract}
Resumen Las mujeres diabéticas con la enfermedad arterial coronaria sometidas a revascularización tienen mayor riesgo de reestenosis debido a las alteraciones fisiopatológicas a nivel vascular propias de la diabetes, a la disminución del efecto protector de los estrógenos después de la menopausia y porque el calibre de sus arterias es menor que el de las no diabéticas y menor que el de los hombres. El objetivo de este artículo es comparar la tasa de reestenosis en la arteria coronaria descendente anterior en las mujeres diabéticas que se sometieron a intervención coronaria percutánea más el stent, frente a la cirugía de revascularización. Para ello se realizó una revisión de la literatura utilizando artículos publicados en bases de datos científicas, que compararan las dos técnicas, haciendo énfasis en la población diabética femenina. Encontrando que hubo una ventaja de la cirugía de revascularización sobre la intervención coronaria percutánea en los eventos de revascularización de la lesión diana (OR 0,253, IC 95\% 0,092-0,703, $\mathrm{p}=0,008)$ y revascularización del vaso diana (OR 0,185, 95\% Cl 0,079 a 0,432, p<0,001), correspondiente a una ventaja en la presentación de evento cardiovascular y cerebrovascular (OR $0,429, \mathrm{Cl} 95 \%$ 0,254-0,723, $\mathrm{p}=0,001)$. Finalmente, se hizo un análisis crítico, concluyendo que la cirugía de revascularización es la técnica de elección en las pacientes diabéticas puesto que tiene una tasa de reestenosis menor y con ello menos eventos adversos que de esta se derivan. (c) 2016 Sociedad Colombiana de Cardiología y Cirugía Cardiovascular. Publicado por Elsevier España, S.L.U. Este es un artículo Open Access bajo la licencia CC BY-NC-ND (http:// creativecommons.org/licenses/by-nc-nd/4.0/).
\end{abstract}

\section{Left anterior descending coronary artery restenosis in diabetic women}

Abstract Women with diabetes and coronary artery disease who undergo revascularization have a higher risk of restenosis due to the pathophysiological alterations at vascular level inherent to diabetes, to the decrease of the protective effect of estrogens after menopause and to the caliber of their arteries being smaller than women without diabetes or men. The goal of this study is to compare the rate of restenosis in anterior descending coronary artery in women

\footnotetext{
* Autor para correspondencia.

Correo electrónico: taniarodriguezc25@gmail.com (T.P. Rodríguez).
} 
Percutaneous

coronary

intervention;

Restenosis with diabetes who underwent percutaneous coronary intervention and the stent, compared to revascularization surgery. For that a literature review was carried out using medical articles published in scientific databases where both techniques were compared, and focusing on female population with diabetes. Results showed there was an advantage for the revascularization surgery versus percutaneous coronary intervention in the revascularization events of the target lesion (OR 0.253, IC 95\% 0.092-0.703, $\mathrm{p}=0.008$ ) and revascularization of the target vessel (OR $0.185,95 \% \mathrm{Cl} 0.079$ a $0.432, \mathrm{p}<0.001$ ), corresponding to an advantage in the presentation of the cardiovascular and cerebrovascular event (OR 0.429, CI 95\% 0.254-0.723, $\mathrm{p}=0.001)$. Finally, a critical analysis was conducted, thus concluding that revascularization is the chosen technique for female patients with diabetes because of the lower restenosis rate, avoiding its adverse events.

(c) 2016 Sociedad Colombiana de Cardiología y Cirugía Cardiovascular. Published by Elsevier España, S.L.U. This is an open access article under the CC BY-NC-ND license (http:// creativecommons.org/licenses/by-nc-nd/4.0/).

\section{Introducción}

Según datos recientes de la OMS en el mundo hay más de 347 millones de personas con diabetes ${ }^{1}$. Esta enfermedad constituye per se un factor de riesgo cardiovascular mayor y se estima que aproximadamente un $44 \%$ de los diabéticos tipo । y un $52 \%$ de los diabéticos tipo ॥ mueren de enfermedad cardiovascular (principalmente cardiopatía y accidente

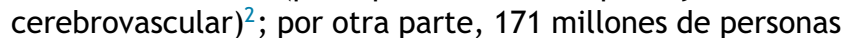
se diagnostican cada año con esta enfermedad; se prevé un incremento de 366 millones $(4,4 \%)$ para el año $2030^{3}$. En Colombia, la prevalencia de diabetes en la población entre 15 y 64 años fue de $4,4 \%$ entre las mujeres y de $3,5 \%$ en hombres, además un $7,9 \%$ presenta riesgo de enfermedad coronaria a 10 años acorde a la ecuación de riesgo cardiovascular de Framingham ${ }^{4}$.

Las mujeres diabéticas tienen más lesiones coronarias en comparación con las no diabéticas ${ }^{5}$. Hay evidencia de que las mujeres con esta enfermedad, experimentan peores resultados que los hombres, independientemente de la edad $^{6}$.

La enorme carga en morbimortalidad ha conducido al desarrollo de numerosas estrategias terapéuticas. Los primeros datos que se conocen del tratamiento quirúrgico de la enfermedad arterial coronaria datan del siglo xx, cuando en el año 1910, Alexis Carrel, padre de la cirugía vascular, anastomosó una arteria innominada de un perro a una arteria coronaria de un segundo perro, para restituir el flujo vascular de este ${ }^{6}$. Vinberg introdujo el implante de la arteria mamaria en el músculo cardiaco en el año 1946. En el año 1966 Kolesov reportó su experiencia inicial con la arteria mamaria ${ }^{7}$. La angioplastia, iniciada por Gruentzig, prometió un enfoque menos invasivo para la revascularización ${ }^{8}$. A partir de ahí, muchas han sido las investigaciones que se han confrontado a través de estudios multicéntricos y aleatorizados en ambas técnicas (cirugía de revascularización vs. intervención coronaria percutánea), con el fin de demostrar cuál de ellas aporta un mayor beneficio para los pacientes en cuestión de pronóstico y calidad de vida.

Esta revisión del tema tiene como objetivo: comparar la intervención coronaria percutánea y la cirugía de derivación o revascularización para establecer cuál de ellas genera menor reestenosis en la arteria coronaria descendente anterior en las pacientes diabéticas sometidas a estos procedimientos y por tanto, un mejor pronóstico de vida a largo plazo.

\section{Materiales y métodos}

Se realizó una revisión del tema basada en los artículos que se encontraron en las bases de datos tales como: PubMed y Science direct utilizando las palabras clave cirugía de revascularización coronaria, diabetes mellitus, stent, intervencionismo coronario percutáneo y reestenosis, seleccionando preferentemente artículos publicados entre los años 2010 a 2016, haciendo énfasis en la población diabética femenina. Los artículos que no incluyeran estos temas no fueron tenidos en cuenta.

\section{Resultados}

\section{Intervención coronaria percutánea vs. la cirugía de revascularización coronaria}

La enfermedad arterial coronaria representa actualmente una importante causa de mortalidad a nivel mundial ${ }^{9}$, razón por la cual los procedimientos de revascularización miocárdica constituyen una importante opción de tratamiento para dicha enfermedad. Durante los últimos años, las indicaciones para la cirugía de revascularización y para la intervención coronaria percutánea, han sido tema de numerosas discusiones e investigaciones, entre las más importantes se encuentra el estudio SYNTAX, que fue un ensayo prospectivo aleatorio multinacional en el que 1.800 pacientes con enfermedad arterial coronaria fueron asignados aleatoriamente a someterse a una intervención coronaria percutánea con los stents liberadores de paclitaxel (PCl: $n=903$ ) o la cirugía de revascularización coronaria (CABG: $n=897)$. De la población total, 452 eran diabéticos y 1.348 no lo eran. Cinco años de seguimiento clínico se completaron en el $86,5 \%$ de los pacientes de la cirugía de revascularización y el $94,5 \%$ de los pacientes de la intervención coronaria percutánea. Dentro de los resultados del estudio se tuvieron en cuenta la necesidad de repetir la 
revascularización por reestenosis después de la intervención coronaria percutánea en comparación con la cirugía, en pacientes no diabéticos y diabéticos ${ }^{10}$. En el año 2013 se publicaron los resultados finales de 5 años de seguimiento del estudio SYNTAX, en el cual el porcentaje de necesidad de repetir la revascularización fue menor en cirugía 14,6\% y en intervencionismo coronario percutáneo $35,3 \%(p<0,001)^{11}$.

El estudio FREEDOM aleatorizó a 1.900 enfermos diabéticos con enfermedad multivaso a recibir un tratamiento revascularizador percutáneo (stents farmacoactivos) o quirúrgico, la edad media fue de 63,1 $\pm 9,1$ años y el $29 \%$ fueron mujeres. Los resultados para las tasas de revascularización repetida en un año en el grupo de intervención coronaria percutánea fue de $12,6 \%$, en comparación con el grupo de bypass coronario 4,8\% (HR: 2,74; IC del 95\% 1,91- 3,89; $\mathrm{p}<0,001)^{12}$.

La revascularización de la arteria coronaria en diabetes (CARDIA) es el nombre del primer ensayo aleatorio controlado realizado únicamente con los pacientes diabéticos ( $n=510 ; 24$ centros) con enfermedad arterial coronaria de múltiples vasos o enfermedad arterial coronaria compleja de un vaso, asignados al azar para intervención coronaria percutánea o cirugía de bypass coronario. Encontrando como resultados que, a un año de seguimiento, la tasa de mortalidad, infarto de miocardio, ictus o nueva revascularización fue de $26 \%$ y 37,5\% (HR: 1,56; IC del 95\% 1,14 a 2,14; $p=0,005)$, respectivamente ${ }^{13}$.

El estudio de las terapias de revascularización arterial (ARTS STUDY) fue un ensayo aleatorio controlado que utilizó 1.205 pacientes asignados al azar para la cirugía de revascularización (605) y para la implantación del stent (600). Dentro del grupo del stent, los pacientes diabéticos tenían una tasa de mortalidad significativamente mayor que los pacientes no diabéticos $(13,4 \%$ vs. $6,8 \%, p=0,03$; RR 1,98 ; IC del $95 \%, 1,11$ a la 3,52) ${ }^{14}$.

La investigación en la revascularización por bypass y angioplastia (BARI) comparó la cirugía por bypass coronario y la angioplastia con balón en 1.829 pacientes con la enfermedad arterial coronaria de múltiples vasos entre los que tenían diabetes. La tasa fue del $25,7 \%$ en el grupo de la intervención percutánea y del $23,1 \%$ en el grupo de la cirugía de revascularización ${ }^{15}$.

Un estudio de la Universidad de Emory (Atlanta), la angioplastia vs. la cirugía de revascularización (EAST) en los pacientes con la enfermedad coronaria de múltiples vasos, arrojó como resultados que la supervivencia a los 8 años es de un $79,3 \%$ en el grupo de angioplastia y $82,7 \%$ en el grupo quirúrgico $(p=0,40)^{16}$.

Algunos metaanálisis como el desarrollado por Aziz y Col., comparan los resultados entre la derivación mínimamente invasiva de la arteria torácica interna a la arteria coronaria y la colocación percutánea de stents como las intervenciones primarias para las lesiones aisladas de la arteria descendente anterior izquierda, incluyeron 12 estudios (1.952 pacientes), seis de ellos eran prospectivos aleatorizados, encontrando que la tasa de la revascularización para la colocación de stents transluminal fue de $13 \%$ en comparación con el 4\% para la cirugía de derivación mínimamente invasiva de la arteria torácica interna a la arteria coronaria descendente anterior izquierda (OR 4,63, IC del 95\% 2,52 a 8,51) ${ }^{17}$.

Otro metaanálisis similar de los ensayos controlados aleatorios en 1.210 pacientes, realizado por Kapoor y Cols., comparó la eficacia de las intervenciones coronarias percutáneas y la cirugía de bypass en la arteria coronaria descendente anterior izquierda proximal, encontrando que la intervención coronaria percutánea se asoció significativamente con una mayor tasa de revascularización repetida que la cirugía de revascularización en un año (RD 14,4\%, IC 95\%: 6,1-22,6 $p=0,001)$; esto siguió siendo significativamente mayor a los cinco años (RD 26,7\%, IC 95\%: 20,1-33,3) ${ }^{18}$.

\section{Consideraciones en las mujeres}

\section{La cirugía de revascularización vs. la intervención coronaria percutánea}

Se realizó un seguimiento a largo plazo de las pacientes sometidas a la intervención coronaria percutánea y mujeres con la cirugía de revascularización para comparar los resultados de las dos técnicas, donde se incluyeron 817 mujeres: $489(59,8 \%)$ fueron sometidas a tratamiento en la arteria descendente anterior con intervencionismo coronario con stents liberadores de fármacos frente a 328 (40,2\%) con la cirugía de revascularización en la misma arteria, de las cuales tenían diabetes 161 pacientes que recibieron intervencionismo y 101 sometidas a la cirugía. El seguimiento clínico se obtuvo a una mediana de 1.185 días (rango intercuartil [RIC] 628 a 1.548). Con respecto a la mortalidad por todas las causas, fue del $14,1 \%$ frente al $7,0 \%$, y la mortalidad por causa cardiovascular fue de $7,0 \%$ frente a $4,6 \%$ en el grupo con la intervención coronaria percutánea frente al grupo con la cirugía de revascularización, respectivamente; con respecto al infarto de miocardio, se reportó tal evento en $4,3 \%$ frente a $1,5 \%$, con la revascularización de la lesión diana $10,2 \%$ frente a $3,8 \%$, y las tasas con la revascularización del vaso tratado de $15,1 \%$ frente a $5,1 \%$. Se encontró el desarrollo del evento cardiovascular y cerebrovascular mayor en $30,5 \%$ frente a $15,7 \%$. En las pacientes con enfermedad distal tratadas con un stent, estos eventos fueron de $28,4 \%$, frente a $35,2 \%$ en aquellas que requirieron dos stent. Además, la trombosis del stent ocurrió solo en 6 de las mujeres $(1,2 \%)$ tratadas con la intervención coronaria percutánea.

Hubo una ventaja de la cirugía de revascularización sobre la intervención coronaria percutánea en los eventos de revascularización de la lesión diana (TLR) (OR 0,253, IC 95\% 0,092-0,703, $\mathrm{p}=0,008)$ y revascularización del vaso diana (TVR) (OR 0.185, 95\% Cl 0,079 a 0.432, $\mathrm{p}<0,001$ ), correspondiente a una ventaja en la presentación de evento cardiovascular y cerebrovascular mayor (OR 0,429, Cl 95\% $0,254-0,723, p=0,001)^{19}$.

\section{La intervención coronaria percutánea}

En un estudio de 4.936 pacientes, donde 1,396 (28,2\%) eran mujeres, 596 fueron tratadas con el stent convencional, donde el $16,9 \%$ de las mujeres tratadas con el stent convencional tenía diabetes y $20,8 \%$ de las mujeres con esta patología fueron tratadas con el stent liberador de fármacos. Habían sido tratadas con el stent convencional en la arteria descendente anterior $56,2 \%$ y con el stent liberador de fármacos $57,6 \%$. Las mujeres tuvieron un mayor riesgo de resultados adversos después de la intervención 
coronaria percutánea que los hombres. En cuanto al impacto del sexo en los resultados tres años después de la intervención con el stent. Estudios como el T-SEARCH y el RESEARCH, demuestran que la revascularización del vaso diana y eventos adversos cardiacos graves fueron significativamente, más bajos en las mujeres tratadas con el stent liberador de fármacos que en las mujeres tratadas con el stent convencional (cociente de riesgos instantáneos para la revascularización del vaso diana (HR): 0,52 [95\% intervalo de confianza (IC): 0,36-0,75], HR ajustado por eventos cardiacos adversos graves: [IC del $95 \% 0,48$ a la 0,83$] 0,63)^{20}$.

\section{La cirugía de revascularización}

Al comparar la cirugía de revascularización con bomba y sin bomba se encontró que la tasa de mortalidad de las mujeres sometidas a la cirugía de revascularización coronaria sin circulación extracorpórea es casi un punto porcentual inferior a la de las mujeres sometidas a cirugía con bomba $(3,12$ vs. 3,$90 ; p=0,052$ ). Las mujeres sometidas a la cirugía de bypass coronario con bomba experimentan una tasa de $42 \%$ mayor mortalidad $(p=0,0239)$ que las mujeres que se someten a cirugía de bypass coronario sin bomba ${ }^{21}$.

\section{Discusión}

\section{Enfermedad arterial coronaria y mecanismos moleculares de reestenosis en diabéticos}

Los pacientes con diabetes mellitus tienen una mayor tasa de estenosis que los pacientes no diabéticos ${ }^{22}$, y desarrollan aterosclerosis coronaria precoz en múltiples vasos ${ }^{23}$. Los factores más importantes que precipitan su aparición, son: la hiperglucemia y el incremento de ácidos grasos libres, producto de la resistencia a la insulina, que desencadenan reacciones inflamatorias mediadas por las citosinas. La hiperglucemia conduce a disfunción endotelial al estimular la producción de metabolitos intermediarios que tienen acción prooxidante como las especies reactivas del oxígeno y causan daño vascular directo. Otra posible fuente de radicales libres en la diabetes es la autooxidación de la glucosa, la cual facilita la generación de cetoaldehídos reactivos y la formación de productos finales de la glicosilación avanzada, los cuales pueden reaccionar con el óxido nítrico e inactivarlo; lo que pudiera explicar, en parte, la disminución de la respuesta vasodilatadora que se observa en las arterias de los individuos diabéticos y la frecuente aparición de hipertensión arterial en estos sujetos. Además, hay un incremento de la actividad de la óxido nítrico-sintetasa, lo que aumenta la producción del anión superóxido, el cual interviene en la formación del compuesto prooxidante peroxinitrito que es capaz de oxidar las lipoproteínas de baja densidad e inactivar a la prostaciclina sintetasa, provocando disfunción endotelial ${ }^{24}$.

Por otra parte, Asakawa y Cols., notaron que los niveles de factor de crecimiento incrementaron notablemente cuando las células endoteliales fueron cultivadas con altos niveles de glucosa, sugiriendo así que las complicaciones microvasculares de la diabetes estarían relacionadas con estos eventos ${ }^{25}$.
Los hallazgos de Fuster y Cols. ${ }^{26}$, en cuanto a las respuestas proliferativas neointimales halladas en las arterias coronarias estenosadas, plantean que el mecanismo más común de reestenosis involucra el desarrollo de la hiperplasia intimal fibrocelular y proliferación de las células musculares lisas, que comienza desde las 24-48 horas después del daño vascular. Kohcki y Cols. ${ }^{27}$, demostraron cambios morfológicos arteriales en los pacientes fallecidos días después de la angioplastia; específicamente, dos días después del procedimiento hay ruptura de la placa ateromatosa, cubierta por capas de plaquetas, fibrina y trombina. Los hallazgos morfológicos 140 días después de la injuria con balón, consisten en la proliferación de las células del músculo liso, intercaladas dentro de un estroma de tejido conectivo denso. Otras investigaciones ${ }^{28}$, también han descrito secuencias similares de eventos, hasta 3 meses después de la angioplastia, lo cual conduce al engrosamiento de la íntima y oclusión vascular.

\section{Principales factores que precipitan la enfermedad coronaria en la población femenina}

\section{Diabetes mellitus}

La tasa de eventos cardiovasculares relacionados con la diabetes se incrementa en frecuencia a edades más tempranas (50-59 años) en la mujer en comparación a los hombres (6069 años $)^{29}$. Además, el riesgo de la mortalidad coronaria aumenta de 3 a 7 veces en comparación con el incremento de 2 a 3 veces al observado en los hombres diabéticos; después de 65 años de edad, una de cada tres mujeres, desarrolla algún tipo de enfermedad cardiovascular. Una de las explicaciones de esto es que los estrógenos inducen un incremento temprano de receptores de lipoproteínas de baja densidad, si el nivel de estrógenos séricos disminuye, los lípidos séricos pueden incrementar. Esto sugiere que los estrógenos pueden contribuir a disminuir los niveles séricos de estas lipoproteínas proporcionando protección cardiovascular, no obstante, este efecto protector se encuentra ausente en la mujer diabética.

Anatómicamente, el calibre medio de las arterias coronarias de los pacientes diabéticos es menor que el de los no diabéticos, sobre todo si es mujer, que en promedio tienen el diámetro de las arterias coronarias $0,30 \mathrm{~mm}$ menor que los hombres ${ }^{30}$. Estas circunstancias, comprometen la permeabilidad a mediano y largo plazo, lo cual está asociado con un mayor riesgo de reestenosis ${ }^{31}$.

Por su parte, la arteria coronaria descendente anterior proximal suministra del 45 a $55 \%$ del flujo de sangre al ventrículo izquierdo. En consecuencia, los eventos isquémicos que se producen en estos segmentos proximales son a menudo fatales ${ }^{32}$, por lo cual la reducción significativa del lumen de estos tiene implicaciones de peor pronóstico ${ }^{33}$.

\section{Factores de riesgo psicosociales en Colombia}

Poco se ha indagado sobre el papel de los factores psicosociales en la etiología de la enfermedad cardiovascular ${ }^{34}$. Por ejemplo, en las mujeres: la depresión y el estrés marital tienen un papel mediador que finalmente resulta en 
la enfermedad coronaria ${ }^{35}$. Además, diferentes estresores: en el hogar, en el trabajo, financieros y los eventos vitales adversos se relacionan como factores de riesgo para infarto del miocardio ${ }^{36}$. El no tener un cubrimiento por parte de las instituciones de salud y ser analfabeta fueron los factores de riesgo sociales más prevalentes para desarrollar la enfermedad cardiovascular grave en Colombia, asimismo, los factores de riesgo psicológico con mayor prevalencia en la población son: el desempleo $(42,5 \%)$, seguido de la carga laboral excesiva $(22,2 \%)^{37}$.

\section{Reestenosis en la arteria coronaria descendente anterior}

Pocos estudios recientes han comparado los resultados de la intervención coronaria percutánea $(\mathrm{PCI})$ con la cirugía de revascularización (CABG) en los pacientes con la enfermedad arterial coronaria en la descendente anterior izquierda aislada, sin embargo, varios metaanálisis como los publicados por Aziz y Cols. y Kapoor y Cols., en los cuales se tenían en cuenta estudios aleatorizados que evaluaban las tasas de reestenosis en la arteria coronaria descendente anterior tanto en $\mathrm{PCl}$ como en $\mathrm{CABG}$, encontrando una mayor tasa de revascularización repetida en $\mathrm{PCI}$ que $\mathrm{CABG}$ en un año.

\section{Cirugía de la revascularización vs. la intervención coronaria percutánea}

En el estudio SYNTAX compararon la necesidad de repetir la revascularización por reestenosis después de la intervención coronaria percutánea y después de la cirugía de revacularización, la importancia de este estudio radica en que evaluaron los desenlaces de dichas técnicas en los pacientes no diabéticos y en diabéticos, dentro de los resultados del seguimiento de los pacientes se incluían la tasa de revascularización repetida el cual fue $20,7 \%$ menor en la cirugía que en el intervencionismo coronario percutáneo. Aunque el estudio FREEDOM evaluó a los enfermos diabéticos con enfermedad multivaso solamente, encontró resultados similares, las tasas de revascularización repetida en un año en el grupo de intervención coronaria percutánea fue de 7,8\% mayor en comparación con el grupo de bypass coronario.

En el ensayo clínico CARDIA las tasas de nueva revascularización fueron significativamente mayores para el grupo de la intervención percutánea; por otra parte, a los cinco años de seguimiento en el mismo estudio se observa un aumento significativo, en la tasa de muerte para el grupo intervenido con el stent, lo que podría implicar mejores resultados a largo plazo para los pacientes intervenidos por la cirugía de bypass.

Las diferencias observadas en el ensayo ARTS para cualquier revascularización a los cinco años es del 21,5\%, para una posterior cirugía es de $9,3 \%$, y para una posterior intervención percutánea es del $14,9 \%$. La diferencia en la tasa de revascularización repetida entre los dos grupos aumenta con el tiempo del $17,2 \%$ en un año a $21,5 \%$ en cinco años sin una diferencia concomitante de la mortalidad en este período de tiempo. Vale la pena aclarar que, a pesar del riesgo adicional de repetición de la revascularización en el grupo con el stent en comparación con el grupo de la cirugía, esto no se tradujo en un aumento de mortalidad. Para el caso de los pacientes diabéticos tratados con el stent, la tasa de eventos adversos cardiacos fue mayor en comparación con los pacientes no diabéticos intervenidos de la misma forma, caso contrario para aquellos que fueron sometidos a la cirugía, puesto que no hubo diferencia importante en la tasa de eventos adversos cardiacos, con una diferencia porcentual de solo un $4 \%$.

\section{Recomendaciones actuales para el intervencionismo coronario en pacientes diabéticos}

Las guías de manejo del año 2011 del Colegio Americano de Cardiología/la Asociación Americana del Corazón y las guías europeas del año 2010 reconocen la importancia de la presencia de la diabetes al momento de decidir sobre la estrategia de revascularización. Las directrices establecen que es razonable optar por la cirugía de revascularización, en particular si un injerto de la arteria mamaria interna izquierda se puede anastomosar a la arteria descendente anterior, en lugar de la intervención coronaria percutánea en pacientes con la diabetes y la enfermedad arterial coronaria de múltiples vasos (clase lla, nivel B); mientras que las guías europeas establecen que la cirugía debe ser considerada en pacientes con la diabetes y la cardiopatía isquémica estable de múltiples vasos cuando el grado de la enfermedad justifica un enfoque quirúrgico, especialmente en pacientes con la enfermedad arterial coronaria de múltiples vasos con un perfil de riesgo aceptable (clase Ila, nivel B). Por otra parte, cuando se utilizan los stents, recomiendan el uso de los stents liberadores de fármacos para reducir la reestenosis (clase I, nivel $\mathrm{A})^{38,39}$.

\section{Cirugía de revascularización e intervencionismo coronario percutáneo en mujeres}

Solo pocos informes han evaluado las estrategias óptimas de la revascularización en mujeres con la enfermedad arterial coronaria, quienes presentan en comparación a los hombres más comorbilidades y características angiográficas desfavorables. No obstante, se encontró una ventaja en la cirugía de revascularización sobre el intervencionismo coronario en cuanto al desarrollo de los eventos cardiovasculares y los cerebrovasculares, pues a largo plazo fueron menores en el grupo de la cirugía.

Hay mejores resultados clínicos después de la intervención coronaria percutánea con el uso del stent liberador de fármacos. No obstante, debido a la falta de representación de las mujeres en los estudios no está claro si los hallazgos se pueden generalizar a la población femenina. Este stent también se asocia con menores tasas de la revascularización del vaso diana y eventos adversos cardiovasculares y cerebrovasculares. La superioridad de los stents liberadores de fármacos para reducir la revascularización se mantiene en ambos sexos hasta 3 años después de la intervención. Las mujeres tratadas con estos stent, presentaban mayor prevalencia de la diabetes que se asocia con mayores tasas de reestenosis.

En cuanto a la cirugía de revascularización, las mujeres permanecen más sintomáticas que los hombres, tienen 
mayor oclusión del injerto y suelen requerir repetidas revascularizaciones ${ }^{40}$.

Las mujeres que se someten a la cirugía de bypass coronario sin bomba tendrían menores tasas de mortalidad que las sometidas a la cirugía de bypass coronario con bomba. Las mujeres con la cirugía de bypass coronario sin bomba tendrían un menor número de complicaciones mayores (trauma/hemorragia, cardiacas) que las mujeres que se someten a la cirugía con bomba.

En la literatura médica son pocos los estudios que se realizan teniendo en cuenta las diferencias entre mujeres y hombres, desafortunadamente, el conocimiento sobre la eficacia y seguridad de las pacientes que se reporta actualmente se deriva principalmente de los análisis de subgrupo de poblaciones del género masculino, predominantemente. Las mujeres tratadas están en mayor riesgo aunque los resultados adversos de las mujeres diabéticas sometidas al stent han disminuido significativamente con el tiempo, todavía tienden a tener peores resultados clínicos que los hombres ${ }^{41}$.

\section{Conclusiones}

- La presencia de la diabetes en las mujeres se asocia con mayores tasas de reestenosis; en ellas, la revascularización de la descendente anterior con la cirugía de bypass se asocia a una mayor incidencia de resultados adversos a corto y mediano plazo. A largo plazo los resultados de muerte o infarto de miocardio son similares para la intervención con stents y cirugía. No obstante, a 5 años las pacientes con los stents requirieron mayores reintervenciones debido a la aparición de reestenosis, lo cual señala a la cirugía de revascularización como la técnica de elección.

- Los resultados clínicos después de la intervención coronaria percutánea con stent liberador de fármacos, demuestran que este se asocia con menores tasas de revascularización del vaso diana y eventos adversos.

- Las mujeres sometidas a la cirugía de bypass coronario sin bomba tendrían menores complicaciones y tasas de mortalidad que las mujeres sometidas a la cirugía de bypass coronario con bomba.

- Una mejor comprensión de los resultados específicos de género permitirá la revascularización individualizada y el desarrollo de estrategias para la gran y creciente población de las mujeres con enfermedad arterial coronaria.

\section{Responsabilidades éticas}

Protección de personas y animales. Los autores declaran que para esta investigación no se han realizado experimentos en seres humanos ni en animales.

Confidencialidad de los datos. Los autores declaran que en este artículo no aparecen datos de pacientes.

Derecho a la privacidad y consentimiento informado. Los autores declaran que en este artículo no aparecen datos de pacientes.

\section{Conflicto de intereses}

Los autores no presentan ningún conflicto de intereses.

\section{Bibliografía}

1. Danaei G, Finucane MM, Lu Y, Singh GM, Cowan MJ, Paciorek $\mathrm{CJ}$, et al. Global Burden of Metabolic Risk Factors of Chronic Diseases Collaborating Group (Blood Glucose). Lancet. 2011;378:31-40.

2. Morrish NJ, Wang SL, Stevens LK, Fuller JH, Keen H. Mortality and causes of death in the WHO Multinational Study of Vascular Disease in Diabetes. Diabetología. 2001;44 Suppl 2:S14-21.

3. Wild S, Roglic G, Green A, Sicree R, King H. Global prevalence of diabetes: estimates for the year 2000 and projections for 2030. Diabetes Care. 2004;27:1047-53.

4. Bautista LE, Oróstegui M, Vera LM, Prada GE, Orozco LC, Herrán OF. Prevalence and impact of cardiovascular risk factors in Bucaramanga, Colombia: results from the Countrywide Integrated Noncommunicable Disease Intervention Programme (CINDI/CARMEN) baseline survey. Eur J Cardiovasc Prev Rehabil. 2006;13:769-75.

5. Gatto N, Liu Hodis H, Mack Wendy CR. Brachial artery vasoreactivity is associated with cross-sectional and longitudinal anatomical measures of atherosclerosis in postmenopausal women with coronary artery disease. Atherosclerosis. 2008;196:674-81.

6. Carrel A. On the experimental surgery of the thoracic aorta and heart. Am J Surg. 1919;52:83.

7. Lobo JG, Leitão MC, Martins F, De Siqueira R, Régis R, Landim R, et al. Total Myocardial Revascularization Without Cardiopulmonary Bypass: A Reality. The Heart Surgery Forum. 2002;5:173-6.

8. BARI Investigators. Comparison of coronary bypass surgery with angioplasty in patients with multivessel disease. N Engl J Med. 1996;335:217-25.

9. Mathers CD, Loncar D. Projections of global mortality and burden of disease from 2002 to 2030. Plos Med. 2006;3:e442.

10. Kappetein P, Head SJ, Morice MC, Banning AP, Serruys PW, Mohr FW, et al. Treatment of complex coronary artery disease in patients with diabetes: 5-year results comparing outcomes of bypass surgery and percutaneous coronary intervention in the SYNTAX trial. Eur J Cardiothorac Surg. 2013;43:1006-13.

11. Mohr F, Morice MC, Kappetein P. Coronary artery bypass graft surgery versus percutaneous coronary intervention in patients with three-vessel disease and left main coronary disease: 5year follow-up of the randomised, clinical SYNTAX trial. Lancet. 2013;381:629-38.

12. Farkouh M, Dangas G, Leon M, Smith C, Nesto R, Buse JB, et al. Design of the Future Revascularization Evaluation in Patients with Diabetes Mellitus: Optimal Management of Multivessel Disease (FREEDOM) Trial. American heart journal. 2008;155:215-23.

13. Randomized comparison of percutaneous coronary intervention with coronary artery bypass grafting in diabetic patients. 1year results of the CARDia (Coronary Artery Revascularization in Diabetes) trial. J Am Coll Cardiol. 2010;55:432-40.

14. Serruys P, Ong A, Lex A, Van Herwerden, Sousa JE, Jatene A. Five-Year Outcomes After Coronary Stenting Versus Bypass Surgery for the Treatment of Multivessel DiseaseThe Final Analysis of the Arterial Revascularization Therapies Study (ARTS) Randomized Trial. J Am Coll Cardiol. 2005;46:575-81.

15. The BARI Investigators. The final 10-year follow-up results from the bari randomized trial. Journal of the American College of Cardiology. 2007;49.

16. King SB, Kosinski AS, Guyton RA, Lembo NJ, Weintraub WS. Eight-year mortality in the Emory Angioplasty versus Surgery Trial (EAST). J Am Coll Cardiol. 2000;35:1116-21. 
17. Aziz O, Rao C, Panesar SS, Jones C, Morris S, Darzi A, Athanasiou T. Meta-analysis of minimally invasive internal thoracic artery bypass versus percutaneous revascularisation for isolated lesions of the left anterior descending artery. BMJ. 2007;334:617.

18. Kapoor J, Gienger A, Ardehali R, Varghese R, Perez M, Sundaram V, et al. Isolated disease of the proximal left anterior descending artery comparing the effectiveness of percutaneous coronary interventions and coronary artery bypass surgery. JACC Cardiovasc Interv. 2008;1:483-91.

19. Buchanan GL. Comparison of Percutaneous Coronary Intervention (With Drug-Eluting Stents) Versus Coronary Artery Bypass Grafting in Women With Severe Narrowing of the Left Main Coronary Artery (from the WomeneDrug-Eluting stent for LefT main coronary Artery disease Registry). American Journal of Cardiology. 2014;113:1348-55.

20. Onuma Y. Impact of Sex on 3-Year Outcome After percutaneous Coronary Intervention Using Bare-Metal and Drug-Eluting Stents in Previously Untreated Coronary Artery Disease. JACC: Cardiovascular Interventions. 2009;2:603-10.

21. Brown P. Outcomes experience with off-pump coronary artery bypass surgery in women. The Annals of Thoracic Surgery. 2002;74:2113-9.

22. Kereiakes DJ, Smits PC, Kedhi E, Parise H, Fahy M, Serruys PW, et al. Predictors of deathor myocardial infarction, ischaemicdriven revascularisation, and major adverse cardiovascular events following everolimus-eluting or paclitaxel-eluting stent deployment: Pooled analysisfrom the SPIRIT II, III, IV and COMPARE trials. Eurolntervention. 2011;7:74-83,.4.

23. Hoffman D, Tranbaugh R. Interventions for Coronary Artery Disease (Surgery vs. Angioplasty) in Diabetic Patients. Endocrinology and Metabolism Clinics of North America. 2014;43:59-73.

24. Naito R, Kasai T. Coronary artery disease in type 2 diabetes mellitus: Recent treatment strategies and future perspectives. World J. Cardiol. 2015;7:119-24.

25. Choi IJ, Park HJ, Seo SM. Predictors of early and late target lesión revascularization after drug-eluting stent implantation. J. Interv. Cardiol. 2013;26:137-44.

26. Ip JH, Fuster V, Israel D, Badimon L, Badimon J, Chesebro JH. The role of platelets, thrombin and hyperplasia in restenosis after coronary angioplasty. J Am Coll Cardiol. 1991;17:77-88.

27. Kohcki K, Takebayashi S, Block PC, Hiroki T, Nabuyoshi M. Arterial changes after percutaneous coronary angioplasty: results at autopsy. J Am Coll Cardiol. 1987; 10:592-9.

28. Ueda M, Becker AE, Fujimoto T. Pathological changes induced by repeated percutaneous transluminal coronary angioplasty. $\mathrm{Br}$ Heart J. 1987;58:653-743.

29. Aronson D, Edelman E. Coronary Artery Disease and Diabetes Mellitus. Cardiol Clin. 2014;32:439-55.

30. Schofer J, Schluter M, Rau T. Influence of treatment modality on angiographic outcome after coronary stenting in diabetic patients: a controlled study. J Am Coll Cardiol. 2000;35:1554-9.

31. González J, Castaño M. Coronary Artery Surgery in Diabetic Patients. Rev Esp Cardiol. 2002;55:1311-22.
32. Costa F, Adamo M, Ariotti S, Ferrante G, Pio Navarese E, Leonardi S, et al. Left main or proximal left anterior descending coronary artery disease location identifies high-risk patients deriving potentially greater benefit from prolonged dual antiplatelet therapy duration. Eurolntervention. 2016;11:e1222-30.

33. Joon C, Jeoung H, Ho S, Han J, Min S, Hee E, Won S. Type 4 dual left anterior descending coronary artery. Korean J Intern Med. 2015;30:727-9.

34. Rosengren A, Hawken S, Ôunpuu S, Sliwa K, Zubaid M, Almahmeed WA, et al. Association of psychosocial risk factors with risk of acute myocardial infarction in 11119 cases and 13648 controls from 52 countries (the INTERHEART study): case-control study. The Lancet. 2004;364:953-62.

35. Balog P, Janszky I, Leineweber C, Blom M, Wamala SP, OrthGomer K. Depressive symptoms in relation to marital and work stress in women with and without coronary heart disease. The Stockholm Female Coronary Risk Study. J Psychosom Res. 2003;54:113-9.

36. Pearson TA, Blair SN, Daniels SR, Eckel RH, Fair JM, Fortmann SP, et al. AHA Guidelines for Primary Prevention of Cardiovascular Disease and Stroke. Circulation. 2002;106:388-91.

37. Sánchez L, Barbosa JM, Arias SA. Morbilidad cardiovascular por autoreporte y su asociación con factores biopsicosociales en el Tolima. Rev. Fac. Nac. Salud Pública. 2014;32:80-7.

38. Wijns W, Kolh P, Danchin N, Di Mario C, Falk V, Folliguet $\mathrm{T}$, et al. Task Force on Myocardial Revascularization of the European Society of Cardiology (ESC) and the European Association for Cardio-Thoracic Surgery (EACTS); European Association for Percutaneous Cardiovascular Interventions (EAPCI). Guidelines on myocardial revascularization. Eur Heart J. 2010;31: 2501-55.

39. Hillis LD, Smith PK, Anderson JL, Bittl JA, Bridges CR, Byrne JG, et al., American College of Cardiology Foundation; American Heart Association Task Force on Practice Guidelines; American Association for Thoracic Surgery; Society of Cardiovascular Anesthesiologists; Society of Thoracic Surgeons. 2011 ACCF/AHA Guideline for Coronary Artery Bypass Graft Surgery: a report of the American College of Cardiology Foundation/American Heart Association Task Force on Practice Guidelines: developed in collaboration with the American Association for Thoracic Surgery, Society of Cardiovascular Anesthesiologists, and Society of Thoracic Surgeons. J Am Coll Cardiol. 2011;58:e123-210.

40. Chieffo A, Hoye A, Mauri F, Mikhail GW, Ammerer M, Grines C, et al. Gender-Based Issues in interventional cardiology: A consensus statement from the women in innovations (WIN) Initiative. Catheterization and cardiovascular interventions. 2010;75:145-52.

41. Ortolani P, Solinas E, Guastaroba P, Casella G, Manari N, Piovaccari G, et al. Long-term clinical outcomes after drug eluting stent implantation in women with de novo coronary lesions Results from the REAL (Registro Regionale AngiopLastiche Emilia-Romagna) multicenter registry. International Journal of Cardiology. 2011;149:55-62. 